\title{
Deutsche in Lateinamerika
}

\author{
von Torsten Eßer
}

\begin{abstract}
Denn die Freiheit ist verloren in dem ganzen Europa, darum Brüder, laßt uns reisen nach Amerika!
\end{abstract}

(Aus einem deutschen Auswandererlied)

\begin{abstract}
„Am 16. Juli 1799 bei Tagesanbruch lag eine grüne, malerische Küste vor uns. Die Berge von Neuandalusien begrenzten, halb von Wolken verschleiert, nach Süden den Horizont. Die Stadt Cumana mit ihrem Schloß erschien zwischen Gruppen von Kokosbäumen. Um neun Uhr morgens, 41 Tage nach unserer Abfahrt von Coruña, gingen wir im Hafen vor Anker." Mit diesen Worten beschrieb Alexander von Humboldt seine Ankunft in Venezuela. Er war nicht der erste Deutsche, der lateinamerikanischen Boden betrat, aber vor allem durch seine Reiseberichte ließen sich viele Aussiedlungswillige beeinflussen, Lateinamerika als Ziel zu wählen.
\end{abstract}

\section{Elend und Krieg - Warum Deutsche auswanderten}

Als 1648 der dreißigjährige Krieg endete, lebten auf dem Gebiet, welches rund zweieinhalb Jahrhunderte später als Deutsches Reich etabliert wurde, schätzungsweise nur noch 13,5 der zu Beginn des Krieges 21 Millionen Menschen. Die Städte waren verwüstet, das Land verheert, und die Not war groß. Zwischen 1655 und 1702 folgten weitere Glaubens- und Erbfolgekriege, die neue Verwüstungen, Hungersnöte und steuerliche Belastungen der Bevölkerung nach sich zogen. So wundert es nicht, daß reine Verzweiflung die Menschen aus "Deutschland“ trieb, oftmals trotz bestehender Auswanderungsverbote.

Im Vergleich zur deutschen Auswanderung in die Vereinigten Staaten kamen viel weniger Deutsche bzw. Deutschsprachige nach Lateinamerika. Qualitativ ist ihr Einfluß jedoch nicht zu unterschätzen, da es sich um Besiedler öder Landstriche handelte oder um vertriebene Eliten. Seit dem 17. Jahrhundert siedelten Deutsche verstärkt in Lateinamerika. Bis dahin handelte es sich um Einzelfälle, die aber auch bedeutend sein konnten, wie das Beispiel des Deutschen Bartholomäus Blümlein (oder Blumen) zeigt, der mit dem spanischen Eroberer Pedro de Valdivia ins heutige Chile kam und $1541 \mathrm{zu}$ den Mitbegründern Santiagos gehörte oder das der 50 sächsischen Bergleute, die mitsamt ihren Frauen auswanderten und in den von den Welsern gegründeten Kupferminen (1528) in Santo Domingo arbeiteten. Im selben Jahr schlossen die Welser einen fünfundzwanzigjährigen Vertrag über die Ausbeutung der Ressourcen des heutigen Venezuela mit der spanischen Krone ab, da Karl V. hohe Schulden bei innen zu begleichen hatte. Von Santo Domingo aus verlagerten die Welser ihre Aktivitäten nach Venezuela und gründeten Maracaibo. Ihre Aktivitäten und der Vertrag endeten nach verlustreichen Expeditionen ins Landesinnere, als der neueingesetzte spanische Gouverneur 1546 Batholomäus Welser mit einer stumpfen Machete köpfen ließ.

Die Autoren Bernecker und Fischer gliedern die deutsche Aus- bzw. Einwanderung in fünf bedeutende Phasen, beginnend mit der europäischen Hungerkrise 1816/17, die einen Einwanderungsschub vor allem nach Brasilien zur Folge hatte. Die europäische Agrarkrise 1846/47 und die gescheiterte Revolution von 1848 lösten die zweite Einwanderungswelle 
aus, während derer fast 23.000 Deutsche nach Lateinamerika kamen. Spitzenwerte erreichte die Zahl der Auswanderer in der dritten Phase (1866-1900) in den Jahren 1885 und 1894, als jeweils ca. 16.500 Auswanderer registriert wurden. Auch 13 Sozialdemokraten waren darunter, die Bismarcks Sozialistengesetz vertrieben hatte und die 1882 den ältesten Arbeiterverein Argentiniens gründeten. Sie gaben auch die Zeitschrift Vorwärts heraus, die später gegen die Nazis eine wichtige Rolle einnahm. Herausgeber der Vorwärts war von 1937 bis 1952 der Kommunist Erich Bunke, dessen Tochter Tamara an der Seite von Ernesto Che Guevara im Kampf in Bolivien fiel. In der Zeit zwischen den beiden Weltkriegen ist die vierte Phase anzusiedeln, in der allein von 1920-24 86.000 Einwanderer aus Deutschland gezählt werden konnten, die verarmt waren. Haupteinwanderungsländer waren während des gesamten Zeitraumes Brasilien und Argentinien, in geringerem Maße auch Uruguay und Chile. Mexiko, die Karibik und Zentralamerika blieben eher unbedeutend.

Besondere Aufmerksamkeit verdient die fünfte Phase: Politische und rassische Verfolgung im „Dritten Reich“ führten von 1939-45 zu einer Flüchtlingswelle von ca. 90.000 Menschen, hauptsächlich aus dem Bildungsbürgertum. Ihnen folgten nach Ende des II. Weltkrieges die NS-Täter. Eine nicht zu bestimmende Anzahl von NS-Verbrechern fand in lateinamerikanischen Ländern, besonders in Argentinien, mit Hilfe der Organisation „Odessa“ Unterschlupf. So wurde 1986 auf dem deutschen Friedhof in Los Polvinos, in der Nähe von Buenos Aires, unter falschem Namen ein Mann zu Grabe getragen, der in Wirklichkeit Walter Kutschmann hieß und dem die Ermordung von 2.500 polnischen Juden angelastet wurde.

Argentinien war das Hauptziel vieler Altnazis, weil die Nazis Buenos Aires zum strategischen Zentrum ihrer Südamerikapolitik mit dem Ziel der „Reorganisation des Deutschtums" ausersehen hatten. Dort existierte seit 1931 eine NSDAP-Ortsgruppe unter Leitung von Willi Köhn gab. Der ehemalige Landesgruppenleiter der NSDAP in Chile konnte schon 1935 nach Berlin telegrafieren, daß die Partei über 2.000 Mitglieder hatte, die meisten deutschen Handelshäuser und Banken von ihr beherrscht wurden und daß ihr mit der La-Plata-Zeitung sogar ein Sprachrohr zur Verfügung stand. Der deutsche Schriftsteller und Kleist-Preisträger Paul Zech, dem, kaum war er von Bord eines Emigrantenschiffes gegangen, schon wieder Braunhemden begegneten, schrieb in seinem Tatsachenroman „Deutschland, dein Tänzer ist der Tod“, daß „unter dem Hakenkreuz 16.000 braunangestrichene Seelen beieinander [waren]", wohlgemerkt in Buenos Aires am 1. Mai 1936. Das Nebeneinander von Verfolgten und Verbrechern des Naziregimes führte schon vor und erst recht nach dem Krieg zu einer Situation, die der Emigrant Balder Olden in der Exilzeitschrift Das Andere Deutschland als „....in einer Umwelt deutsche Welt und deutsche Unwelt“ bezeichnete. Es gab zwei Tageszeitungen, zwei Theater usw. Erst die Zeit heilte diese Risse langsam.

Seit Ende der fünfziger Jahre setzte die „normale“ Auswanderung nach Lateinamerika wieder ein. Vor allem das verstärkte Engagement deutscher Unternehmen, das von den Lateinamerikanern sehr positiv gewertet wird, zieht Deutsche an. Allerdings machten Deutsche auch wieder negative Schlagzeilen: 1961 wurde die aus verschiedenen Gründen mysteriöse Colonia Dignidad gegründet. Ihr Gründer, der pädophile Sektenprediger Paul Schäfer, war Anfang der sechziger Jahre mit ca. 300 Anhängern aus Siegburg nach Chile gekommen, um diversen Prozessen zu entgehen. Nch dem Ende der Militärdiktatur kamen nach un nach seine Verbrechen ans Licht, er konnte aber erst 2005 in Argentinien verhaftet werden.

Ein Phänomen der deutschen Besiedlung ist, daß die meisten Siedler sich gegenüber einer kulturellen und sozialen Einflußnahme durch die Gesellschaften der Gastländer abschotteten. Das gilt besonders für die ländlichen Besiedler. Sie versuchten vielmehr, die alte Heimat so getreu wie möglich nachzubilden. Baustil, Kleidung soziale und 
konfessionelle Einrichtungen blieben so wie die Siedler sie kannten. Sie nutzten die alte Heimat und die damit verbundenen Gepflogenheiten als Vorbild, um den neuen Raum wirtschaftlich zu erschließen und waren damit oft sehr erfolgreich. Kultur und Wertehaltung blieben jedoch streng abgetrennt von denen der Einheimischen. Erst nach dem II. Weltkrieg ist in vielen Ländern, zum Beispiel in Chile, ein Ablösungs- und Entfremdungprozeß festzustellen, der zur Assimilation der Deutschen führte oder noch führt. Besonders gut kann man diese Entwicklung an der venezolanischen Siedlung Tovar erkennen, die 1843 von südbadischen Siedlern gegründet wurde. Sie liegt in einem Hochtal $120 \mathrm{~km}$ weit von der karibischen Küste entfernt. Als die ersten Siedler nach zweimonatiger Überfahrt und mehrtägigem Fußmarsch in diesem Tal ankamen, waren sie entsetzt über den Zustand der Umgebung. Trotzdem rafften sie sich auf, bauten eine Siedlung und machten den Boden fruchtbar. Sie lebten abgeschieden, das erste motorisierte Fahrzeug erreichte die Siedlung 1948. Erst ab 1963 gab es eine asphaltierte Straße zum Ort. Dann allerdings setzte eine rasante Entwicklung ein, die den Ort zum touristischen Anziehungspunkt, vor allem der caraqueños, werden ließ, da die deutsche Dorfidylle mit den weißen Fachwerkhäusern und die deutsche Küche etwas Exotisches in Venezuela darstellen, wobei die althergebrachte Kultur nicht mehr gelebt, sondern nur noch vorgeführt wird.

\section{Deutsche Bauern und Eliten}

Bei den ersten Auswanderern handelte es sich um organisierte Gruppen, die sich als Agrarsiedler u.a. in den Cono Sur-Ländern niederließen. Sie gründeten u.a. São Leopoldo und Blumenau in Brasilien. Sie siedelten im noch nicht kultivierten Urwald und betrieben dort intensive Landwirtschaft. Diese Kleinbauernkolonien trugen zur Erhöhung der Wirtschaftskraft und zur Verbesserung der Versorgung der Städte mit Nahrungsmitteln bei. Durch hohe Geburten- und Zuwanderungsraten aufgrund ausreichender Erträge expandierten die Siedlungen, die in dieser Form erst von den europäischen Einwanderern nach Lateinamerika gebracht wurden. Noch heute kann man eine ähnliche Entwicklung bei den deutschstämmigen Mennoniten im Urwald von Belize beobachten.

In Brasilien, dessen erste Beschreibung (1557) dem Deutschen Hans Staden zu verdanken ist, erregten der relative Wohlstand und die Privilegien der deutschen Siedlungen bald die Mißgunst der Großgrundbesitzer, so daß sich zu den ohnehin großen Opfern, die die Siedler erbrachten, noch Schwierigkeiten mit den Nachbarn und Behörden gesellten. Die erste große Besiedlungswelle (1824) ist einer gezielten Werbeaktion im Auftrag des brasilianischen Kaisers Dom Pedro I. zu verdanken. Bis 1939 kamen dann ca. 75.000 deutsche Siedler in die unbsiedelten Gebiete Südbrasiliens, wo heute noch 1/5 der Gesamtbevölkerung deutschstämmig ist. Hier liegen auch die Wurzeln des brasilianischen Ex-Umweltministers José Lutzenberger. In ihren Rodungssiedlungen entstanden soziale Einrichtungen wie Schulen und Schützenvereine, die auch dazu dienten, die Identität der Gruppe gegenüber der brasilianischen Gesellschaft zu schützen. Selbst die Endogamie wurde häufig auch um den Preis der physischen Degeneration beibehalten.

Den Venezolanern sind vor allem die deutschen Generäle und Legionäre in Erinnerung, die an der Seite Simón Bolívars die Freiheit der Kolonien erkämpften. Namen wie Lützow (Luzón) oder Uslar sind heute noch vielen Venezolanern bekannt.

Nach Chile wanderten zwischen 1840-1914 ca. 20.000 Deutsche aus. Städte wie NeuBraunau oder Peñaflor verdanken ihnen ihre Gründung. Die Einwanderung ist wesentlich der Initiative Bernhard Eunom Philippis zu verdanken, der als Seeoffizier die Küste und das Landesinnere Chiles zwischen 1831 und 1837 erforscht hatte und den Plan faßte, diese Gebiete mit deutschen Einwanderern zu kolonisieren. Er überzeugte die Regierung von dieser Idee und wurde später zum Einwanderungsbevollmächtigten ernannt. Unter 
ihm und seinen Nachfolgern werden Osorno, Valdivia und Umgebung und der Raum um den Llanquihue-See besiedelt. Da es sich meistens um abgelegene Gebiete handelte, genossen die Einwanderer in Chile eine relativ hohe Autonomie. Es entwickelten sich eine Selbstverwaltung in gewissen Bereichen (Schule, Kirche) sowie ein von den traditionellen Handelszentren Valparaiso und Santiago unabhängiger Kapitalismus. Die argentinische Regierung schickte deutsche Siedler nach Patagonien. Doch erst zwischen 1920-31 kamen die meisten Deutschen nach Argentinien (47.000). Die Mehrzahl siedelte sich als Handwerker oder Händler im Großraum Buenos Aires an.

Von größerer qualitativer Bedeutung war die Elitenwanderung. Kaufleute und Unternehmer ließen sich zeitweise in den städtischen Ballungsräumen an der Küste nieder, um den Agrar- und Rohstoffexport nach, und den Industriegüterimport von Europa zu kontrollieren. Viele dieser jungen Männer stammten aus den protestantischen Händlerfamilien der Städte Hamburg und Bremen. Sie kamen in der Absicht, in die Heimat zurückzukehren, blieben jedoch oft und gründeten ihr eigenes Geschäft. In Mexiko waren zum Beispiel um 1820 30\% aller seßhaften Deutschen Händler oder Handelsgehilfen. Vor allem durch den Pioniergeist deutscher Kaufleute und durch von deutschen Bankiers geförderte Infrastrukturprojekte (Eisenbahn, Häfen), wurden immer neue Regionen erschlossen. So erschloß zum Beispiel Carlos Gesell, ab 1931 einen öden Sandstreifen an der Küste Argentiniens, das Gebiet des heutigen Villa Gesell, einer Ferienstadt 400 km südöstlich von Buenos Aires, mit mehr als 100.000 Badegästen jährlich.

Während der Zeit des Nationalsozialismus war der Wanderungsstrom weitgehend durch jüdische Fluchtwanderung bestimmt. Zwischen 70.000 und 80.000 Juden kamen in dieser Zeit nach Lateinamerika. Argentinien, Brasilien und Chile als Haupteinwanderungsländer gingen zu einer restriktiveren Einwanderungspolitik über, als ab 1938 die Flüchtlingszahlen anschwollen, während andere Staaten erst dann ihre Grenzen öffneten, zum Beispiel Bolivien, Mexiko, Kuba oder die Dominikanische Republik.

Viele Staaten, bzw. deren Beamte, machten allerdings auch gute Geschäfte mit der Not der Emigranten. So mußten hohe Bestechungsgelder für falsche Pässe, Visa oder Berufsbezeichnungen gezahlt werden. Wer keinen „verwendbaren“ Beruf hatte, wurde zurückgewiesen. Der größte Teil der immigrierten Juden kam jedoch aus den begüterten Schichten und hatte eine entsprechende Ausbildung genossen. Das Geistes-, Kultur- und Wirtschaftsleben der Einwanderungsländer hat in großem Ausmaß von diesen Menschen und ihren Fähigkeiten - mit Ausnahme der Nazis - profitiert. Ob die deutsche Einwanderung nach Lateinamerika insgesamt zu einer Bereicherung des Kontinents beigetragen hat, dafür lassen sich genausoviele Pro- wie Contra-Argumente finden. Unbestritten ist allerdings das Verdienst deutscher Forschungsreisender wie Alexander von Humboldt oder Teobert Maler, deren Arbeiten viel zum Verständnis des Kontinents beitrugen.

\section{Kaffeeunternehmer in Costa Rica}

Die deutsche Einwanderung nach Zentralamerika und in die Karibik war zahlenmäßig wesentlich geringer als in andere Regionen Lateinamerikas. Ungeachtet dessen gingen wichtige, vor allem wirtschaftliche Impulse, von den deutschen Siedlern aus. In Nikaragua war Luis Elster 1850 der erste, der Kaffee kultivierte, was inm viele deutsche Siedler in Guatemala nachmachten, und K.A. Frank gehörte zu den Deutschen, die um 1870 in Panama die ersten Bananenplantagen anlegten.

In Costa Rica gehörten viele deutsche Siedler und gehören heute viele Deutschstämmige zu den reichen und einflußreichen Kreisen des Landes. Dieser Umstand ist u.a. dem Kaffee zu verdanken. Einer der ersten - dokumentierten - Deutschen, die sich in Costa Rica niederließen, war 1826 Georg Stiepel. Er zeichnet dafür verantwortlich, daß ab 1832 
Kaffee nach Chile und von dort aus nach Europa exportiert wurde. So legte er auch den Grundstein für die florierende Kaffeeindustrie, die zur Achse und Säule der costarikanischen Wirtschaft wurde und vor allem viele Deutsche reich machte. Erleichtert wurde diese Entwicklung durch die Tatsache, daß England und Deutschland im 19. Jahrhundert die Hauptabnehmerländer für Kaffee waren.

Noch heute stößt man in Costa Rica auf die Namen und Nachfahren der reichen Kaffeebarone. Familien wie Rohrmoser, nach der ein Stadtteil in San José benannt ist, Peters, Niehaus oder Steinvorth, von der, wie sich beim 125jährigen Einwanderungsjubiläum 1996 herausstellte, mehr Nachfahren (450) in Costa Rica als in Deutschland leben, beeinflussen noch heute das politische und gesellschaftliche Leben im Land. Durch geschickte Heiratspolitik verbanden sie sich mit den spanischen Eliten oder verschafften sich durch ihre Leistungen Zugang zu den Hebeln der Macht: Sie trieben den Bau der Eisenbahn voran, gründeten die erste Brauerei des Landes, wie in vielen anderen lateinamerikanischen Ländern auch, sowie 1912 das Colegio Humboldt - noch heute eine der besten Schulen des Landes - und sie stellen und stellten einflußreiche Politiker, wie Außenminister Niehaus (1980) oder Ferdinand Streber, der es nach der Gründung des Amtes für Statistik, in den siebziger Jahren des vorigen Jahrhunderts bis zum Kriegsminister brachte.

Eines der ersten großangelegten Siedlungsprojekte in Costa Rica hängt mit dem Namen von Bülow zusammen. Dieser deutsche Baron war von der Idee besessen, eine deutsche Kolonie in Costa Rica zu gründen. 1848 riefen er und andere Deutsche die Sociedad Berlinesa de Colonización ins Leben und erwarben ein großes Stück Siedlungsland zwischen Turrialba und der Atlantikküste. Durch die instabile politische Lage in Deutschland entschlossen sich viele dem Auswanderungsaufruf zu folgen. Doch die Unternehmungen standen unter einem schlechten Stern. Zwischen 1851-53 fanden eine Reihe von Reisen statt, in deren Verlauf viele Auswanderer während der Schiffspassage an Krankheiten oder auf dem beschwerlichen Landweg - damals noch über Nikaragua - an den Strapazen starben. Auch befanden sich nicht genug Bauern unter den Siedlern, so daß die Landnahme sich schwierig gestaltete. Als dann schließlich 1856 der U.S.amerikanische Abenteurer William Walker mit seinen Truppen aus Nikaragua ins Land einfiel, um ganz Zentralamerika unter seine Kontrolle zu bringen, es verwüstete, und so eine Choleraepidemie ausgelöst wurde, der auch von Bülow zum Opfer fiel, war das Projekt gescheitert. Doch von den verbliebenen Deutschen entwickelten sich viele zu einflußreichen Plantagenbesitzern, so die Familie Rohrmoser. Des weiteren ging aus diesem Krieg die erste deutschsprachige Zeitung Zentralamerikas, La Gaceta Alemana, hervor, mit der Absicht, die Welt über diesen Krieg zu informieren. Auch wenn während des II. Weltkriegs viele Deutschstämmige aufgrund ihrer deutsch-nationalen Einstellung das Land verließen, um für ihr Vaterland zu kämpfen, so blieb doch die Mehrheit in ihrer neuen „Heimat“ und ist, wie die Buchhandlung Lehmann oder die Kaffeedynastie Peters im costarikanischen Alltag allgegenwärtig.

Literaturtipps:

Bernecker, Walther L./ Thomas Fischer. „Deutsche in Lateinamerika“, in: Klaus J. Bade (Hrsg.). Deutsche im Ausland - Fremde in Deutschland: Migration in Geschichte und Gegenwart, München 1992, S. $197-214$. Eisenbürger, Gert. Lebenswege - 15 Biographien zwischen Europa und Lateinamerika, Hamburg 1995. Embajada de la República Federal de Alemania en Costa Rica. Alemania y Costa Rica, San José 1996. Fröschle, Hartmut (Hrsg.). Die Deutschen in Lateinamerika, Tübingen 1979.

Herrera Balharry, Eugenio. Los Alemanes y el Estado Cafetalero, San José 1988.

Houwald, Güetz von. Los Alemanes en Nicaragua. Pioneros y Emprendedores (www.manfut.org). Hucke, Matthias. „'Wir sind wahre Nationalsozialisten.' Die deutsche Kolonie auf Kuba 1933-1944“, in: Ottmar Ette/ Martin Franzbach (Hrsg.). Kuba heute, Frankfurt a.M. 2001, S. 801-828. 
Kellenbenz, Hermann/ Schneider, Jürgen: La emigración alemana a américa latina desde 1821 hasta 1930. In: Jahrbuch GSWG Lateinamerikas 13/1976, S.386-403.

Liga Chileno-Alemana (Hrsg.). Die Deutschen und die deutsch-chilenische Gemeinschaft in der Geschichte Chiles, Santiago de Chile 2001.

Matices. Heft Nr. 15/ 1997 „Deutsche in Lateinamerika“ (mit Artikeln zur Auswanderung und zu Brasilien, Kuba, Mexiko, Argentinien und Zentralamerika).

Mybes, Fritz. Die Geschichte der aus der deutschen Einwanderung entstandenen lutherischen Kirche in Chile, Düsseldorf 1993.

Rosenberg, Peter. Deutsche Minderheiten in Lateinamerika [Sprache], Frankfurt Oder 2001

(http://viadrina.euv-frankfurt-o.de).

Schonebohm, Dieter. „Alemanes, Judíos y Judíos Alemanes en el Uruguay de los Años 1920 y 1930 “, in:

Jahrbuch für Geschichte Lateinamerikas Nr. 35/ 1998, S. 219-238.

Schrader, Achim. „Deutsche Einwanderung nach Brasilien“, in: Dietrich Briesemeister et al. (Hrsg.). Brasilien heute, Frankfurt a.M. 1994, S. 603-621.

Weil, Jorge. Frutillar: pasado y presente. 150 años de la colonización alemana de Llanquihue, Valdivia 2002. 